\title{
Physical Connections Between Pulsar Outer Magnetospheres and Pulsar Nebulae
}

\author{
Tzihnong Chiueh \\ Physics Department, National Taiwan University, Taipei, Taiwan
}

\begin{abstract}
The spin-down luminosity together with the morphology of pulsar nebula, such a the Crab, have long been known to imply that the pulsar wind be practically unmagnetized immediately before it impinges onto the nebula. In fact, the condition of low wind magnetization can be shown to further imply that the pulsar outer magnetosphere be strongly distorted, where the toroidal field exceeds the conventionally-assumed dipole field by nearly two orders of magnitude in the outer magnetosphere; the current needed requires the plasma density to exceed the GoldreichJulian charge density by several orders of magnitude. Such a physical condition can certainly impose a constraint on the candidate mechanisms of non-thermal radio emission, and may also place a constraint on the high-energy, electrode-gap emission mechanisms.
\end{abstract}

\section{Introduction}

The pulsar wind magnetization is characterized by a dimensionless quantity: the ratio of the Poynting flux to kinetic-energy flux, $\sigma$. Near the light-cylinder radius, $R_{L}$, the plasma is strongly magnetized by a field at least as strong as the dipole magnetic field, of order $f e w \times 10^{5}$ gauss for a Crab-like pulsar, where $\sigma \geq$ $10^{2}$. On the other hand, from the observed nebula expansion, which accounts for the dominant spin-down luminosity, it has been recognized that the pulsar wind at a distance of about 0.1 pc from the star must be practically unmagnetized, with $\sigma_{\infty} \leq 10^{-2}$ (Rees \& Gunn 1974). The fact that the pulsar wind is able to continuously extract energy from its driving source, electromagnetic (EM) fields, to such a degree where the EM energy is depleted almost completely, that has, for decades, posed a challenge for both observers and theorists.

Past proposals for solving this problem center around having the EM energy almost entirely dissipated into the plasma internal energy, which then gets converted into the flow kinetic energy in the wind zone (Kennel, Fujimura \& Okamoto 1983; Coroniti 1990). However, the difficulty pertaining to such proposals arises, among others, from the exceedingly strong high-energy emission, which has not been detected. Different avenues in pursuit of the same subject have been on the line of dissipationless acceleration. A recent work in this direction has verified that it is only near $R_{L}$ can such wind acceleration occur (Chiueh, Li \& Begelman 1998). Though the details of this acceleration mechanism is yet available, certain crucial requirements have been established. First, the poloidal fields must be severely bunched prior to the rapid wind acceleration 
which blows open the bunched fields, thereby depleting most of the EM energy; the expansion factor of the solid angle should be as large as $1 / \sigma_{\infty}$. Second, the toroidal field must much exceed the poloidal field in the bunched fields. Third, once most of the EM energy is converted into the flow kinetic energy, the poloidal fields outward will become nearly radially directed. Fourth, only a small fraction of the magnetospheric field lines, of order $\sqrt{\sigma_{\infty}}$, take part in the pulsar wind. The first and second conditions indicate the operation of the de Laval nozzle mechanism, and the third condition implies little electric current available in the wind for any electromagnetic interaction with plasmas.

\section{Physical connections through the radially directed wind}

The four conditions provide the needed links for extracting the informations of the under-resolved pulsar magnetosphere. The spin-down luminosity has been evaluated from the nebula energy budget and from the pulsar spin-down rate $\dot{P} / P$, which can then be equated to the Poynting flux for an estimate of the field strength at $R_{L}$. Conventionally one assumes a dipole-magnetosphere and a significant fraction of the dipole field lines becoming open to participate the wind acceleration, and this permits a further estimation of the pulsar surface field. However, with the new picture available, we may interpolate the poloidal field strength back to $R_{L}$ with accuracy through the radial field-line geometry. It turns out that the bunched poloidal field $B_{p}$ near $R_{L}$ must be a factor $1 / \sqrt{\sigma_{\infty}}$ greater than the dipole field, i.e., $B_{p}\left(R_{L}\right) \geq$ few $\times 10^{6}$ gauss for a Crab-like pulsar, in order to provide the same energy output. Moreover, the domination of the toroidal field $B_{t}$ requires $B_{t}\left(R_{L}\right) \geq 10^{7}$ gauss. Finally, though this estimate applies only to the wind-loaded field lines, a small fraction of all, the magnetosphere should also consist of a highly distorted field of this magnitude in order to keep the cross-field forces balanced.

Due to the severe distortion of the magnetosphere, it is not a simple matter to determine the surface field strength. Nevertheless, it is safe to assert that the surface field strength should at least be as large as that estimated from the dipole configuration, though a factor of 10 higher is not unreasonable. Furthermore, the plasma density ought to be several orders of magnitude higher than the Goldreich-Julian density to provide a sufficiently large magnetospheric current for field distortion. Both will have an impact on the non-thermal radio-emission mechanisms, in that the radio-wave amplifiers should be able to also operate across the field lines. Finally, it remains to be examined whether the electrodegap models of high-energy emission may survive or not, with a dense plasma and the distorted field lines wound several times around before reaching $R_{L}$.

\section{References}

Chiueh, T., Li, Z.Y., \& Begelman, M.C. 1998, ApJ, 505, 835

Coroniti, F.V. 1990, ApJ, 349, 538

Kennel, C.F., Fujimura, .S., \& Okamoto, I. 1983, Geophys.

Astrophys. Fluid Dyn. 26, 147

Rees, M.J., \& Gunn, J.E. 1974, MNRAS 167, 1 